\title{
Clinical Utility of Selinexor/Dexamethasone in Patients with Relapsed or Refractory Multiple Myeloma: A Review of Current Evidence and Patient Selection
}

This article was published in the following Dove Press journal: OncoTargets and Therapy

\section{Panagiotis Malandrakis** loannis Ntanasis- Stathopoulos (D)* Maria Gavriatopoulou Evangelos Terpos (ID)}

Department of Clinical Therapeutics, School of Medicine, National and Kapodistrian University of Athens, Athens, Greece

*These authors contributed equally to this work
Correspondence: Evangelos Terpos Department of Clinical Therapeutics, School of Medicine, National and Kapodistrian University of Athens, Alexandra General Hospital, 80 Vas. Sofias Avenue, Athens II528, Greece Tel +30-2/3-216-2846

Fax +30-2|3-2|6-25II

Email eterpos@med.uoa.gr

\begin{abstract}
Multiple myeloma (MM) is one the most common hematological malignancies, and despite the survival prolongation offered by proteasome inhibitors (PIs), immunomodulatory drugs (IMiDs) and anti-CD38 monoclonal antibodies, the need for novel agents is prominent. Selinexor is a first-in-class, oral, selective inhibitor of exportin-1 (XPO1), a vital protein for the exportation of more than 200 tumor suppressor proteins from the nucleus. Both in solid tumors and hematologic malignancies, selinexor-mediated inhibition of nucleus export seems to effectively lead to cancer cell death. Selinexor in combination with dexamethasone (Sd) received an accelerated FDA approval on July 2019 for heavily pretreated patients with relapsed/refractory MM (RRMM) based on the promising results of the Phase II STORM trial. The preliminary results of the randomized Phase III BOSTON trial have shown a $47 \%$ increase in progression-free survival among PI-sensitive, RRMM patients who received selinexor with bortezomib-dexamethasone compared with bortezomib-dexamethasone alone. Several different selinexor-containing triplet regimens are currently being tested in the RRMM setting in an umbrella trial, and the preliminary results seem promising. Furthermore, the addition of selinexor in other anti-myeloma agents seems to overcome drug-acquired resistance in preclinical studies. The main toxicities of selinexor are gastrointestinal disorders and hematologic toxicities (mainly thrombocytopenia); however, they are manageable with proper supportive measures. In conclusion, selinexor is a new anti-myeloma drug that seems to be effective in patients who have no other therapeutic options, including patients who have received novel cellular therapies such as CAR-T cells. Its potential role earlier in the therapeutic algorithm of $M M$ is currently under clinical investigation.
\end{abstract}

Keywords: selinexor, exportin, selective inhibitor of nuclear export, relapsed/refractory, myeloma

\section{Introduction}

Multiple myeloma (MM) is an incurable hematological malignancy and is characterized by end-organ damage (anemia, renal failure, bone disease, hypercalcemia) and/or other myeloma defining events. ${ }^{1}$ Treatment advances including proteasome inhibitors (PIs), immunomodulatory drugs (IMiDs) and anti-CD38 monoclonal antibodies have significantly improved the prognosis of patients 
with MM during the last years, whereas autologous stem cell transplant (ASCT) remains a standard option for fit patients. ${ }^{1,3}$ Novel agents are introduced constantly in the therapeutic armamentarium with anti-BCMA antibodies and bispecific antibodies being the most promising., 4 Nevertheless, the survival curve of patients with relapsed/refractory (RR) disease is not flattened, since the vast majority of $\mathrm{MM}$ patients will eventually become refractory to all available agents. For this patient group, the choice is either palliative care or the administration of novel agents with distinct mechanisms of action. In this context, selinexor has been developed to address this unmet therapeutic need.

\section{Biological Rationale and Preclinical Data on Selinexor}

Selinexor (XPOVIO, formerly KPT-330) is a first-inclass, oral, highly specific, slowly reversible, covalent small molecule inhibitor of exportin-1 (XPO1) or chromosome maintenance protein 1 (CRM1), which is an important nuclear exporter for more than 200 nuclear cargo proteins, including many tumor suppressor proteins (TSPs). The overexpression of this protein in myeloma cancer cell lines provides the rationale for applying this new oral selective inhibitor of nuclear exportation (SINE) to suppress the exportation of the TSPs in myeloma cells. As a results, the high concentration of TSPs in the nucleus ultimately leads to cell cycle arrest and apoptosis of the myeloma cells, ${ }^{7,8}$ without affecting the normal cells. ${ }^{9}$ Although XPO1 inhibition affects all XPO1 cargo proteins bearing a nuclear export signal, cancer cells are mainly affected by nuclear export inhibition. This makes nuclear transport receptors promising targets for therapeutic intervention. ${ }^{10}$ The anticancer activity of XPO1 inhibitors seems to have a broad spectrum, since it is p53 mutation independent, which is a common cytogenetic aberration in myeloma cells of patients with RRMM. ${ }^{11}$ Moreover, in vitro and ex vivo data show that XPO1 inhibition disrupts the 3D nuclear organization of telomeres of the chromosomes, which are vital for chromosomal stability especially in cancer cells, whereas normal cells are not susceptible to this effect. $^{12}$

XPO1 is considered to play a key role in the nuclear export of cargo proteins from the nuclear pore to the cytoplasm, including some major (proto-) oncoproteins and tumor suppressors such as BRCA1, p53, cyclin D1. The overexpression of CRM1 has been associated with poor prognosis and adverse clinical outcomes, since it affects nuclear export processes in such a way resulting in inactivation or aberrant activation of cancer-related proteins and, thus, rendering cancer cells insensitive to apoptotic and antiproliferative signals. ${ }^{11,13}$ CRM1 overexpression seems to play an important role in tumor size, cell proliferation and survival in many solid tumors (osteosarcoma, pancreatic cancer, ovarian cancer, cervical cancer, lung cancer) and in chronic lymphocytic leukemia. ${ }^{11}$ Importantly, increased drug-resistance and decreased progression-free (PFS) and overall survival (OS) have been associated with XPO1 overexpression. ${ }^{10,13}$

Regarding MM, the high expression of CRM1 has been associated with myeloma-related bone disease and plays an important role in the survival of $\mathrm{MM}$ cells. ${ }^{11}$ Osteoclastogenesis is a cardinal feature of myelomainduced bone disease and it is orchestrated by NF-kB activation through the receptor activator of nuclear factor $\mathrm{kB}$ ligand (RANKL) and NFAT1c. SINEs inhibit NF- $\kappa B$ activation by RANKL and NFAT1c, prevent the activation of osteoclasts, and impede osteoclastogenesis. ${ }^{14,15}$ The inhibition of CRM1 activity by SINEs seems to affect the intracellular cascade in various ways, by enhancing the caspase-3/7 activity and the cleavage of Poly (ADP-ribose) polymerase (PARP) and caspase-3. SINEs seem to be effective in MM cells with mutated p53, which indicates that the mechanism of action is p53independent, and, at least partially, it could be explained by the blockage of NF- $\kappa$ B. NF- $\kappa$ B seems to play an important role in drug resistance and adhesion of $\mathrm{MM}$ cells to the healthy bone marrow cells. An interesting in vitro finding is that SINEs lead to apoptosis of more than $80 \%$ of MM cells, however, they spare the healthy cells of the bone marrow. Furthermore, the SINE-induced inhibition of NF- $\kappa B$ seems to reduce cytokines like IL-6, VEGF, MIP-1 $\beta$ and IL-10 in the microenvironment, which are vital for the survival of myeloma cells. ${ }^{11}$

Cancer cells have the metabolic flexibility to survive and proliferate in low oxygen tension conditions. XPO1 is associated with the nuclear export of the transcription factor hypoxia-inducible factor (HIF). HIF-1 is a very important factor in the metabolism of cancer cells by mediating the adaptation to hypoxia, and, ultimately, cell survival under hypoxic conditions. Accordingly, higher levels of HIF-1 expression in the nucleus have been correlated with tumor resistance and poor patient prognosis. ${ }^{10}$ XPO1 seems to be upregulated in MM cells 
resistant to bortezomib. Interestingly, the addition of selinexor seems to counteract the hypoxia-induced bortezomib resistance in vitro. The combination of these drugs seem to affect the protein homeostasis leading to protein overload, and eventually increase cell stress and lead to cell death. ${ }^{16}$

Glucocorticoids are a common denominator for MM treatment, and they act by binding to the glucocorticoid receptor (GR) in the cytoplasm, creating a complex that acts as transcription factor. ${ }^{8}$ In preclinical trials, it has been observed that the addition of a glucocorticoid to selinexor intensifies the activity of that complex in nucleus and also increases the transcription level of the GR, thus enhancing the anti-myeloma activity of the glucocorticoid. ${ }^{17}$ Furthermore, the combination of selinexor and dexamethasone ( $\mathrm{Sd})$ seems to impair the mTOR activity, which is a key molecule in myeloma progression, in both GRdependent and GR-independent pathways. Sd upregulates the expression of REDD1, a negative regulator of the mTOR pathway, and REDD1 levels could be used to predict the response to treatment. In vivo xenograft models have confirmed the in vitro findings, since selinexor with dexamethasone have shown a synergistic reduction of tumor growth. ${ }^{18}$

\section{Clinical Evidence}

\section{Early Phase Clinical Trials}

In the Phase I clinical trial (NCT01607892) evaluating selinexor among patients with RRMM and Waldenstrom's macroglobulinemia, the drug was administered with or without dexamethasone. The dosage that was administered varied from $3 \mathrm{mg} / \mathrm{m}^{2}$ to $60 \mathrm{mg} / \mathrm{m}^{2}$ once to three times per week, in order to assess the safety and efficacy of the regimen, and to identify the maximum tolerated dose (MTD) and the recommended Phase 2 dose (RP2D). The overall response rate (ORR) [partial response (PR) or better] was $10 \%$ ( 8 out of 84 patients) [95\% confidence interval $(\mathrm{CI}): 0.05-0.18$ ], with a median duration of response of 5 months (range 2-11). Minimal response (MR) was achieved in $15 \%$ of all patients. Four percent of the patients in the monotherapy arm achieved PR, without any complete remission, and $18 \%$ reached MR. Selinexor at $45 \mathrm{mg} / \mathrm{m}^{2}$ twice weekly (BIW) along with $20 \mathrm{mg}$ of dexamethasone led to an ORR of $50 \%$ (6 out of 12 patients), but the higher dose at $60 \mathrm{mg} /$ $\mathrm{m}^{2}$ was not well tolerated and, surprisingly, it did not result in any significant response (13\% achieved MR).
Thus, the RP2D was set at $45 \mathrm{mg} / \mathrm{m}^{2}$, which is equivalent to a flat dose of $80 \mathrm{mg}$, in combination with $20 \mathrm{mg}$ of dexamethasone. ${ }^{8}$

\section{The STORM Study; A Pivotal Phase 2 Clinical Trial Main Findings}

The pivotal, multicenter, open-label, phase 2 STORM study (part 1) included 79 patients, who were refractory to at least two IMiDs (lenalidomide, pomalidomide) and two PIs (bortezomib, carfilzomib) (quadrefractory patients), along with a subgroup that was also refractory to the anti-CD38 monoclonal antibody daratumumab (penta-refractory patients). The dose administered was as recommended from the phase I trial, $80 \mathrm{mg}$ of selinexor along with $20 \mathrm{mg}$ dexamethasone on days 1, 3, 8, $10,15,17,22,25$ (twice weekly) of each 28-day cycle. The primary endpoint of ORR was 21\% (95\% CI: $13 \%$ to $31 \%)$, although not statistically significant $(\mathrm{P}=0.17)$, with a median duration of response at 5 months (one patient with at least 8.4 months). Regarding the subgroup analysis, the ORR was $21 \%$ for quadrefractory patients and $20 \%$ for penta-refractory patients. Among 17 patients with high-risk cytogenetic abnormalities, the ORR was $35 \%$. The median PFS and OS were 2.3 and 9.3 months, respectively. ${ }^{7,19}$

Based on these results, the phase 2b STORM study was expanded to include 122 penta-exposed, triple-class refractory patients (patients who had received bortezomib, carfilzomib, lenalidomide, pomalidomide, daratumumab, glucocorticoids, and an alkylating agent), 53\% of whom had high-risk cytogenetic abnormalities. The patients received $80 \mathrm{mg}$ of selinexor along with $20 \mathrm{mg}$ of dexamethasone twice weekly in each 4-week cycle, until disease progression, discontinuation due to toxicity, or death. The ORR (partial response or better) was $26 \%$ (95\% CI: 19 to $35 \%$ ), whereas two patients achieved minimal residual disease (MRD) negativity. The study achieved the primary endpoint of an ORR above $10 \%$. The median duration of response was 4.4 months $(95 \%$ CI, 3.7 to 10.8$)$. The median PFS was 3.7 months $(95 \%$ CI, 3.0 to 5.3), and the median OS was 8.6 months (95\% CI, 6.2 to 11.3). Patients who achieved a PR or better, or a minimal response or better, had a median OS of 15.6 months. ${ }^{19,20}$ The clinical benefit rate was $39.2 \%$, and based on these analysis it has been supported that 
any response (MR or better) prolonged OS among these heavily pretreated patients. ${ }^{17}$

Based on these results, selinexor (in combination with dexamethasone) received accelerated approval in the USA in July 2019 for the treatment of adult patients with RRMM who have received at least four prior antimyeloma therapies and whose disease is refractory to at least two PIs, at least two IMiDs and an anti-CD38 monoclonal antibody. ${ }^{7}$

\section{Subgroup Analyses}

The treatment effect on specific subgroups of patients is of particular importance in terms of treatment individualization. Elderly and frail patients necessitate a fine balance of treatment effectiveness and toxicity management, ${ }^{21}$ whereas the treatment of patients with renal impairment is rather challenging. ${ }^{22}$

A post-hoc analysis of the STORM study categorized the patients in three age groups $(<60,60-70,>70$ years old) and showed that patients derive benefit from the treatment with selinexor regardless of age. All age groups had similar ORR, PFS and OS, and similar AEs, although treatment discontinuation was observed more frequently and pneumonia was more common in the age group including patients above 70 years old. ${ }^{21}$

Another post-hoc analysis of the STORM study compared the safety and efficacy of Sd among patients groups with different baseline renal function based on creatinine clearance $(\mathrm{CrCl})(<40,40-60,>60)$. The ORR was similar across subgroups: $35.7 \%, 16.0 \%$ and $28.0 \%(\mathrm{P}=0.35)$, whereas dose reduction $(67 \%, 56 \%, 54 \%)$ or discontinuation $(40 \%, 28 \%, 33 \%)$ were not affected by the presence of lower $\mathrm{CrCl}$ at baseline. Furthermore, $25-67 \%$ of the patients showed an improvement in renal function, based on an increase in $\mathrm{CrCl}$ during treatment with $\mathrm{Sd}^{19}$

\section{Biomarker Analysis}

XPO1 RNA levels isolated from CD138+ bone marrow cells and blood samples of the patients before initiating treatment with selinexor, along with the levels of the glucocorticoid receptor at the nucleus, were suggested as pharmacodynamic biomarkers possibly predicting the response to treatment with Sd. A preliminary analysis revealed a four-master-gene signature, evaluating the expression of IRF3, ARL2BP, ZBTB17, and ATRX genes, that was highly predictive of response to treatment. ${ }^{17,20}$ Further studies in this field in independent patient series are needed in order to validate these findings.

\section{Adverse Events}

The most common non-hematological adverse events reported in the STORM trial were gastrointestinal disorders including fatigue (73\%, grade $3: 25 \%)$, nausea (72\%, grade 3: $10 \%)$, anorexia (56\%, grade 3: $5 \%$ ), diarrhea $(46 \%$, grade $3: 7 \%)$ and vomiting $(38 \%$, grade 3: $3 \%$ ). Grade 3 or 4 hematological toxicities were reported quite often with $58 \%$ of the patients showing thrombocytopenia, $44 \%$ anemia and $21 \%$ neutropenia. The dosage of the regimen was delayed or reduced due to an adverse event in $80 \%$ of the patients, whereas $18 \%$ had to interrupt treatment due to toxicities. ${ }^{17}$ Three patients with no dose limiting toxicities following the first cycle of treatment received a higher dose of selinexor at $100 \mathrm{mg}$ twice weekly, but adverse events led to dose reductions in all three of them. ${ }^{19}$ Thrombocytopenia (43\%), fatigue (16\%) and neutropenia $(11 \%)$ were the most common toxicities that led to dose modifications. ${ }^{19}$ Selinexor-associated thrombocytopenia was not attributed to the deregulation of mature megacaryocytes or platelets. The inhibition was considered to be at the level of early megacaryopoiesis, as selinexor seems to affect the maturation of progenitor cells to megacaryocytes, which are less affected by selinexor as they mature. Importantly, thrombocytopenia was reversible with thrombopoietin-receptor agonists and dose modifications. ${ }^{23}$

\section{Selinexor-Based Combinations with Other Anti-Myeloma Agents Preclinical Evidence}

Although the FDA has approved selinexor for the treatment of RRMM, its ability to restore the sensitivity to other anti-myeloma agents and overcome drug resistance has provided the rationale of combining it with these agents. In preclinical studies, selinexor has shown synergistic activity with other regimens that are already widely used in the treatment of MM. ${ }^{20,24,26}$

Selinexor seems to restore sensitivity to melphalan, which could be explained by the retention of TP53 in the nucleus, the lower levels of $\mathrm{NF \kappa B}$, and the decreased levels of DNA repair proteins FANCF and FANCL of the Fanconi anemia/BRCA pathway. XPO1 inhibition increases the melphalan-induced DNA damage by impairing the DNA repairing mechanisms in myeloma cells. ${ }^{27}$ 
Moreover, XPO1 inhibitors may enhance the antimyeloma activity of doxorubicin. In preclinical models of anaplastic thyroid carcinoma, the combination of selinexor with doxorubicin seems to block the exportation of TOP2A from the nucleus, which is one of the main mediators of doxorubicin-resistance in MM cells, thus, the combination promotes DNA damage and enhances the activity of doxorubicin. ${ }^{28}$

$\mathrm{I} \kappa \mathrm{B} \alpha$ is a tumor suppressor that dimerizes with $\mathrm{NF} \kappa \mathrm{B}$, inhibits transcription, and induces MM cell apoptosis. XPO1 inhibitors along with PIs (bortezomib) result in a higher increase of I $\mathrm{B} \alpha \alpha$ levels compared with monother-

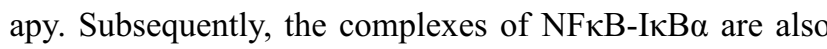
increased, thus, the combination further deactivates $\mathrm{NF} \kappa \mathrm{B} .{ }^{27}$

The addition of selinexor to the widely used PI carfilzomib seems to induce myeloma cell apoptosis and autophagy both in in vitro models and xenograft models. A possible underlying molecular pathway of this effect is the synergic activation of caspase-10. In the XPO1/PI inhibition setting, caspases 10 and 8 colocalize with p62 and create an aggregate that seems to induce a chain reaction resulting in fulminant caspase activation and autophagy. ${ }^{29}$

\section{Clinical Data}

Based on these data, the multicenter Phase $1 b / 2$, multiarm, open-label umbrella Selinexor and Backbone Treatments of Multiple Myeloma Patients (STOMP) trial in RRMM patients (NCT02343042) was conducted in order to assess the MTD and the RP2D of Sd in combination with various widely used anti-myeloma drugs, including bortezomib, carfilzomib, lenalidomide, pomalidomide and daratumumab. Other ongoing studies evaluating selinexor-based triplet regimens in RRMM include the phase 1 SINE study evaluating the combination of Sd with carfilzomib (NCT02199665), the phase 2 study SELIBORDARA evaluating the combination of Sd with daratumumab and bortezomib (NCT03589222) and the randomized Phase 3 Boston trial evaluating the combination of $\mathrm{Sd}$ with bortezomib (NCT03589222). Selinexor is being also evaluated as a conditioning regimen before ASCT in a phase $1 / 2$ study.

\section{Selinexor-Bortezomib-Dexamethasone}

Sd with bortezomib at $1.3 \mathrm{mg} / \mathrm{m}^{2}$ (SVd) was evaluated in the STOMP trial including 42 patients with a median age of 64 years. The participants had a median of 3 prior lines of therapy (range 1-11), and 17\% had high- risk cytogenetics. Half of the patients were refractory to a prior PI and $45 \%$ were refractory to both a PI and an IMiD. The RP2D (24 patients) was set at selinexor $100 \mathrm{mg}$ once weekly, bortezomib at $1.3 \mathrm{mg} / \mathrm{m}^{2}$ once weekly and dexamethasone $40 \mathrm{mg}$ weekly. At the RP2D, there were reported no grade 3 or higher nausea and vomiting events, and the main reasons for discontinuation and dose reduction were grade 3 fatigue (23\%) and grade 3-4 thrombocytopenia (31\%). For the non-PI refractory subgroup the ORR was $84 \%$ (11\% CR), the clinical benefit rate (CBR) was 95\% and the median PFS was 17.8 months. Regarding the PI refractory patients, the ORR was $43 \%$ (5\% CR), the CBR was $67 \%$ and the median PFS was 6.1 months. In the interpretation of these results, it should be noted that a meta-analysis has shown that the ORR of bortezomib retreatment among bortezomib-refractory patients has been estimated at $22 \% .{ }^{30}$ Overall, the median PFS was 9 months for all 40 patients and the time to response was 1.2 months. ${ }^{25}$

Importantly, the primary results of the randomized phase 3 BOSTON study were recently presented. ${ }^{31,32}$ Four hundred and two patients with RRMM and one to three prior lines of therapy were randomized to receive either SVd or Vd. Selinexor was administered at $100 \mathrm{mg}$ once weekly, bortezomib at $1.3 \mathrm{mg} / \mathrm{m}^{2}$ once weekly and dexamethasone at 40mg once weekly. Crossover to SVd upon progression on Vd was allowed. The primary study endpoint was met by showing a 4.47 month (47\%) increase in the median PFS of patients receiving SVd compared with those receiving $\mathrm{Vd}$ (13.93 versus 9.46 months, respectively, $p=0.0066$ ). ${ }^{32}$ SVd was also associated with a significantly higher ORR $(76.4 \%$ vs $62.3 \%, p=0.0012)$, whereas no new safety signals emerged. ${ }^{32}$

\section{Selinexor-Carfilzomib-Dexamethasone}

The efficacy of Sd in combination with carfilzomib (SKd) was evaluated in the phase 1 SINE trial (NCT02199665). At the time of the analysis, a total of 21 patients had been enrolled with a median of 4 prior lines of therapy, whereas $95 \%$ had received carfilzomib and $81 \%$ were dual-class refractory (PI and IMiD) and previously exposed to bortezomib, carfilzomib, lenalidomide and pomalidomide. The RP2D was set at $60 \mathrm{mg}$ of selinexor on days $1,3,8,10,15,17$, carfilzomib at $20 / 27 \mathrm{mg} / \mathrm{m}^{2}$ on days $1,2,8,9,15,16$ and $20 \mathrm{mg}$ of dexamethasone on days $1,2,8,9,15,16,22,23$ ( $10 \mathrm{mg}$ from cycle 5 afterwards) on a 28 - 
day cycle. The CBR was $71 \%$, the ORR was $48 \%$ and the median PFS and OS for all enrolled patients were 3.7 and 22.4 months, respectively. The findings were consistent for the carfilzomib-refractory and the high-risk patient subgroups. The most common grade 3 or 4 toxicities included thrombocytopenia (71\%), anemia (33\%), neutropenia (33\%) and infections $(24 \%) .{ }^{33}$ Promising results have been also reported from the SKd arm in the STOMP trial, where selinexor was administered at 80 or $100 \mathrm{mg}$ weekly, carfilzomib at $56 \mathrm{mg} / \mathrm{m}^{2}$ or $70 \mathrm{mg} / \mathrm{m}^{2}$ on days $1,8,15$ and dexamethasone at $40 \mathrm{mg}$ weekly in a 28 -day cycle. The enrolled patients had a median age of 70 years, they had received a median of 4 prior lines of therapy and all of them were carfilzomib naïve. The ORR was at $72 \%$, whereas 4 patients achieved $\mathrm{CR}$, and the toxicity profile was as consistent with the SINE trial, whereas the most common dose limiting toxicity was thrombocytopenia. ${ }^{34,36}$

\section{Selinexor-lxazomib-Dexamethasone}

A phase 1 trial included 18 heavily pretreated MM patients with median 5 prior lines of therapy (range: 1-11). The patients received selinexor at two different dose levels, once or twice weekly, ixazomib on days 1,8,15 and dexamethasone on the days of selinexor administration (SId). The once weekly schedule was preferred due to better tolerability, and the MTD was determined at $80 \mathrm{mg}$. The ORR was $22 \%$, and the maximum duration of response was 14 months. The most common non-hematologic AEs were gastrointestinal including nausea (50\%), vomiting (33\%), diarrhea (22\%) and anorexia (28\%). For this reason, all patients received prophylactic ondansetron and the addition of olanzapine $2.5 \mathrm{mg}$ was also considered. The most common hematologic AEs were thrombocytopenia in $72 \%$ of the patients (grade 3-4 in 11 patients), anemia in $61 \%$ and neutropenia in $28 \%$. Treatment discontinuation due to gastrointestinal toxicities was quite often, and so the study did not proceed with to the expansion phase. In conclusion, it was suggested that SId is an all-oral regimen that may be applicable to less heavily pretreated patients, in order to avoid thrombocytopenia and severe toxicity resulting in dose delays and reductions. ${ }^{37}$

\section{Selinexor-Lenalidomide-Dexamethasone}

Selinexor, lenalidomide, and dexamethasone (SRd) was tested in a phase $\mathrm{Ib} / \mathrm{II}$ trial among patients who had received at least 1 prior line of therapy and the RP2D was set at $60 \mathrm{mg}$ of selinexor weekly, dexamethasone $40 \mathrm{mg}$ weekly and lenalidomide $25 \mathrm{mg}$ on days $1-21$ of each 28-day cycle. A total of 24 patients were enrolled, whereas 20 were evaluable for response at the time of analysis and 13 received the RP2D. Two patients discontinued treatment due to toxicities $(8 \%)$. The median age was 67 years (range 49-84) and the median number of prior treatments was 1 (range 1-8). The most frequent grade 3 or higher AEs were thrombocytopenia (63\%) and neutropenia (63\%). The ORR and the CBR were both at $92 \%$ for lenalidomide-naïve patients and $13 \%$ and $38 \%$, respectively, for t he group of patients who had been previously exposed to lenalidomide. Overall, the median PFS was 10.3 months, whereas it had not been reached for lenalidomide-naïve patients, and it was 2.8 months for lenalidomide-exposed patients. These data suggest that SRd is effective for patients with RRMM, who have not been previously exposed to lenalidomide. ${ }^{38}$

\section{Selinexor-Pomalidomide-Dexamethasone}

Selinexor-dexamethasone along with pomalidomide (SPd) has been evaluated in 51 patients with a median of 4 prior lines of therapy. Selinexor was administered in two dosing levels $(60 \mathrm{mg}$ and $80 \mathrm{mg}$ ) once or twice weekly, pomalidomide was administered at an escalated dose (2 mg, $3 \mathrm{mg}, 4 \mathrm{mg}$ on days 1-21) along with a low dose of dexamethasone of $20 \mathrm{mg}$ twice weekly or $40 \mathrm{mg}$ weekly. The R2PD was set at $60 \mathrm{mg}$ of selinexor weekly, $4 \mathrm{mg}$ of pomalidomide on days 1 to 21 and $40 \mathrm{mg}$ of dexamethasone weekly of each 28-day cycle. The ORR among pomalidomide-naive patients was $56 \%$ with 19\% achieving VGPR, whereas the ORR among pomalidomide refractory patients was $30 \%$. The median PFS was 12.2 and 5.6 months for pomalidomide-naïve and pomalidomide-refractory patients, respectively. It has to be noted that these response rates are higher than the published data of pomalidomide and dexamethasone alone in the literature showing an ORR of $31 \%$ and a median PFS of less than 4 months. The CBR was $78 \%$ in the pomalidomide-naive group and $74 \%$ among all patients. SPd showed a safe toxicity profile with less than $2 \%$ of patients experiencing grade $3-4$ nausea, vomiting, diarrhea and anorexia, which suggests that these AEs may be dose and schedule dependent. $^{34,35}$ 


\section{Selinexor-Daratumumab-Dexamethasone}

Daratumumab is a CD38 targeting monoclonal antibody and is considered a promising new agent in the treatment of MM. ${ }^{3,39}$ Daratumumab has been evaluated along with Sd (SDd) in a phase Ib study among PI/ IMiD refractory but daratumumab and selinexor naïve patients. Selinexor was administered either biweekly at $60 \mathrm{mg}$, or weekly at $100 \mathrm{mg}$, daratumumab at the standard dose of $16 \mathrm{mg} / \mathrm{kg}$ iv as per standard schedule and dexamethasone at $20 \mathrm{mg}$ biweekly or $40 \mathrm{mg}$ weekly. The dose of selinexor at $100 \mathrm{mg}$, with daratumumab and dexamethasone at $40 \mathrm{mg}$ weekly was considered to be the RP2D. The ORR was $77 \%$, which may be considered superior to the corresponding response rates of daratumumab monotherapy or $\mathrm{Sd}^{25}$

\section{Selinexor-Doxorubicin-Dexamethasone}

Despite the promising preclinical findings, the addition of doxorubicin to $\mathrm{Sd}$ did not improve the ORR (ORR at $15 \%$ ), despite being well tolerated, in a multicenter, openlabel phase I/II clinical trial (NCT02186834). ${ }^{40,41}$

\section{Selinexor After Previous Treatment with Cellular Therapies}

Chimeric antigen receptor (CAR) T-cells constitute a new promising therapeutic approach for RRMM patients, and currently several CAR T-cells constructs targeting various epitopes such as BCMA, CD19, CD38, CD138 are being evaluated in clinical studies. ${ }^{6,42,43}$ CAR T-cell efficacy is quite promising, such as the bb2121 CAR T-cell, that resulted in an ORR of $85 \%$ and a median PFS of 11.8 months among 33 previously treated MM patients. ${ }^{44}$ Among selinexor trials with available results, seven patients received selinexor-based treatment (1 Sd, $1 \mathrm{SVd}, 5 \mathrm{SKd})$ after progression on CAR T-cell therapy. All of them were heavily pretreated with a median of 10 prior lines of treatment, 4 of them were pentarefractory and had rapidly progressive disease. The responses to selinexor-based regimens were a stringent $\mathrm{CR}$, three VGPRs, two PRs and a MR. These data suggest that as cellular therapies are used also in earlier lines of MM therapy, selinexor-based regimens still offer an alternative therapeutic choice probably due to the distinct mechanism of action. ${ }^{45}$

\section{Evaluation of Selinexor in Other Malignancies}

In a phase I dose escalation clinical trial, selinexor was administered to 79 patients with relapsed/refractory non-
Hodgkin lymphoma of different subtypes. The ORR was $31 \%$ for the whole study population, and responses were observed in many NHL subtypes. The RP2D was $35 \mathrm{mg} /$ $\mathrm{m}^{2}$ (approximately $60 \mathrm{mg}$ ) twice weekly. ${ }^{46}$

In another phase I trial, selinexor was administered in various solid tumors and its efficacy was evaluated. Among 157 patients evaluable for response, one patient had a complete response (melanoma), and six patients had radiological partial responses (melanoma, colorectal cancer, ovarian, prostate cancer, thymoma, and cervical cancer). Forty three percent of the patients had stable disease. Among patients with colorectal carcinoma, a greater percentage of the patients with stable disease had the KRAS mutation ( $44 \%$ versus $21 \%$ wild type RAS group), but it was not enough to suggest a predictive role according to the mutational status of KRAS. The RP2D was set at $35 \mathrm{mg} / \mathrm{m}^{2}$ (approximately $60 \mathrm{mg}$ fixed dose) twice weekly. ${ }^{47}$ Based on these results, selinexor was also used in heavily pretreated patients with gynecologic malignancies (ovarian, cervical and endometrial) in a phase II clinical trial. The results showed an ORR of $15 \%$ (all PR), and a disease control rate of $46 \%$. The once weekly dose at $80 \mathrm{mg}$ had a better safety profile than the twice weekly dose at $60 \mathrm{mg}$, whereas the toxicity profile was similar as reported in the myeloma trials. ${ }^{48}$

\section{Management of Toxicities}

In order to evaluate the toxicity profile of the different selinexor-based combinations in $\mathrm{MM}$, an integrated retrospective pool analysis including 437 patients with MM from the phase 1 SINE study (NCT01607892) $(\mathrm{N}=81)$, the STORM study (NCT02336815) $(\mathrm{N}=202)$, the STOMP study (NCT02343042) $(\mathrm{N}=117)$, and the BOSTON clinical trial (NCT03110562) $(\mathrm{N}=37)$ trials was conducted. The median age of all patients was 64 years and $69 \%$ of them have received 5 or more prior lines of therapy. Two thirds of the patients received selinexor on a twice-weekly schedule, $27 \%$ once weekly and $6 \%$ on another schedule. The starting dose was $<60 \mathrm{mg} /$ dose for $21 \%$ of the patients, $61-80 \mathrm{mg} /$ dose for $51 \%$, $81-100 \mathrm{mg}$ for $25 \%$ of the patients and $>100 \mathrm{mg}$ for $3 \%$ of the patients (median weekly dose of $100 \mathrm{mg}$ ). ${ }^{49}$

The most common hematologic AE was thrombocytopenia, and it was observed in $66 \%$ of all patients (any grade), whereas it was grade 3 in $22 \%$ of them and grade 4 in $32 \%$. The experts suggest that the platelet count should be checked weekly in order to monitor potential thrombocytopenia, whereas twice weekly monitoring is 
advised for grade 3 thrombocytopenia. If platelets are below $25.000 / \mu \mathrm{L}$, transfusion was considered in order to avoid dose interruption. In several cases, platelet stimulation growth factors were applied in order to reduce the need for infusions, although this was an off-label indication. Thrombopoietin-receptor agonists (TPORAs) romiplostim ( $1 \mu \mathrm{g} / \mathrm{kg}$ up to $10 \mu \mathrm{g} / \mathrm{kg}$ once weekly) and eltrombopag (50mg po daily) were used in 48 patients with platelet count below $25.000 / \mu \mathrm{L}$, and, interestingly, $67 \%$ of them restored their platelet count in a median time of 14 days. These agents were more effective among patients receiving selinexor once rather than twice weekly. Two other effective approaches in restoring thrombocytopenia were the delay of treatment and dose reductions. However, in some cases the low platelet count persisted even for 7-14 days after the dose modification. Overall, dose reductions were performed in $32 \%$ of the patients due to thrombocytopenia and the most common was from $80 \mathrm{mg}$ biweekly to $100 \mathrm{mg}$ weekly.

Moreover, 29\% of the patients experienced grade 3 or higher neutropenia, whereas $4 \%$ showed febrile neutropenia and $19 \%$ presented with severe infections. The use of growth colony stimulating factors (GCSF) filgrastim and pelfigrastim were used in $75 \%$ of the patients presenting with neutropenia. In the vast majority of them (90\%) neutropenia resolved with a median time to resolution of 8 days.

Nausea is another common AE among patients treated with selinexor with an overall incidence of $68 \%$, whereas it was grade 3 or higher in $6 \%$. The incidence of vomiting was $37 \%$. Therefore, it is recommended that all patients receive a 5-HT3 antagonist (ondansetron $8 \mathrm{mg}$ po twice daily) before the first dose and the following day of selinexor administration for at least 8 weeks. If nausea persists other drugs should be considered like olanzapine (2.5-10 $\mathrm{mg}$ po in the evening), neurokinin 1 (NK1) receptor antagonists like rolapitant $(180 \mathrm{mg})$, aprepitant (80mg) or fosaprepitant $(150 \mathrm{mg})$, benzodiazepines (lorazepam 0.5-1 mg daily) and cannabinoids (dronabinol 2.5-5 mg twice daily). If the drug is then well tolerated, the physician should strongly consider reducing or interrupting the supporting treatment after 8 weeks of treatment. Diarrhea was observed in $41 \%$ of the patients (grade 3 in 5\%) and it was commonly associated with weight loss and other gastrointestinal disorders. The use of loperamide $4 \mathrm{mg}$ po and then $2 \mathrm{mg}$ as needed or bismuth subsalicylate led to diarrhea resolution in $87 \%$ of the patients.

Another common AE was fatigue since it occurred in $63 \%$ of the patients ( $16 \%$ grade 3$)$, whereas it did not resolve in $70 \%$ of them. It is important to acknowledge the multifactorial background of fatigue including the general condition of the patient and the disease status. The administration of methylphenidate $10 \mathrm{mg}$ po daily could be considered. Decreased appetite and weight loss was observed in $53 \%$ of the patients (7\% grade 3 ), and the use of megestrol, cannabinoids, mirtazapine and olanzapine are considered possible therapeutic options. The proper hydration of the patient (at least 2 liters daily), the addition of high-calorie supplements and a general nutritional consult are also important to mitigate this toxicity.

Although the underlying mechanism is unclear, approximately one third of all the patients (32\%) had hyponatremia, mostly asymptomatic (95\%), whereas $19 \%$ presented grade 3 or higher hyponatremia. The use of sodium chloride tablets or intravenous electrolytic fluids corrected the hyponatremia in $83 \%$ of the patients. Pseudohyponatremia could not be ruled out due high paraprotein levels and hyperglycemia. ${ }^{45,49}$

In the STORM trial, an assessment of the quality of life (QoL) of the patients showed a possible worsening in physical well being and functional well-being; however, it can not be exclusively attributed to the drug regimen, taking into consideration that the trial included heavily pretreated patients with RRMM. Future trials should carefully assess QoL-related indices using validated instruments of patients reported outcomes. ${ }^{50}$ In each case, the overall patient clinical status should be taken into consideration including comorbidities and frailty in order to offer a tailored therapeutic approach. ${ }^{51,52}$

\section{Conclusions and Future Perspectives}

Selinexor-based regimens provide a novel approach to patients with RRMM (Table 1). Sd is particularly important for patients with no other therapeutic choices, including those previously treated with novel cellular treatments. The addition of selinexor to other backbone treatments has also shown promising results, whereas the SVd regimen has shown significant clinical activity in a randomized phase 3 study. Patient selection is necessary for determining the optimal selinexor-based 
Table I Summary of Findings of Important Clinical Trials with Selinexor-Based Regimens

\begin{tabular}{|c|c|c|c|c|c|c|c|c|c|}
\hline NCT No & Study & Phase & Regimen & $\mathbf{N}$ & $\begin{array}{l}\text { Median } \\
\text { (Range) } \\
\mathbf{N}^{\circ} \text { of } \\
\text { Prior } \\
\text { Lines }\end{array}$ & ORR & $\begin{array}{l}\text { PFS } \\
\text { (Median, } \\
\text { Months) }\end{array}$ & $\begin{array}{l}\text { OS } \\
\text { (Median, } \\
\text { Months) }\end{array}$ & Toxicities $(\geq 10 \%)$ \\
\hline NCT023368I5 & $\begin{array}{l}\text { STORM } \\
\text { Part I }\end{array}$ & II & Sel-Dex & 79 & $7(3-17)$ & $21 \%$ & 2.3 & 9.3 & $\begin{array}{l}\text { Thrombocytopenia (59\%), } \\
\text { anemia ( } 28 \%) \text {, } \\
\text { neutropenia ( } 23 \%) \text {, fatigue } \\
(15 \%)\end{array}$ \\
\hline NCT023368I5 & $\begin{array}{l}\text { STORM } \\
\text { Part } 2\end{array}$ & II & Sel-Dex & 122 & $7(3-18)$ & $26 \%$ & 3.7 & 8.6 & $\begin{array}{l}\text { Thrombocytopenia (59\%), } \\
\text { anemia }(44 \%) \text {, } \\
\text { hyponatremia }(22 \%) \text {, } \\
\text { neutropenia }(21 \%) \text {, } \\
\text { nausea }(10 \%)\end{array}$ \\
\hline \multirow[t]{4}{*}{ NCT02343042 } & STOMP & $\mathrm{lb} / \mathrm{II}$ & $\begin{array}{l}\text { Sel-Dara- } \\
\text { Dex }\end{array}$ & 34 & $3(2-10)$ & $69 \%$ & $\begin{array}{l}\text { Not } \\
\text { reached }\end{array}$ & NR & $\begin{array}{l}\text { Thrombocytopenia }(42 \%) \\
\text { anemia }(29 \%) \text { leukopenia } \\
(26 \%) \text { neutropenia } \\
(23 \%) \text { lymphopenia }(13 \%) \\
\text { fatigue }(16 \%) \text { hyponatremia } \\
(13 \%)\end{array}$ \\
\hline & & & $\begin{array}{l}\text { Sel-Vel- } \\
\text { Dex }\end{array}$ & 42 & $3(I-I I)$ & $63 \%$ & 9 & NR & $\begin{array}{l}\text { Thrombocytopenia }(50 \%) \\
\text { neutropenia }(26 \%) \text {, anemia } \\
(19 \%) \text {, fatigue }(14 \%)\end{array}$ \\
\hline & & & $\begin{array}{l}\text { Sel-Pom- } \\
\text { Dex }\end{array}$ & 45 & $4(2-9)$ & $50 \%$ & 10.4 & NR & $\begin{array}{l}\text { Neutropenia }(56 \%) \\
\text { thrombocytopenia (3I\%) } \\
\text { anemia ( } 31 \%) \text { leukopenia } \\
\text { (16\%) lymphopenia } \\
(13 \%) \text { febrile neutropenia } \\
(13 \%) \text { fatigue (II\%) }\end{array}$ \\
\hline & & & $\begin{array}{l}\text { Sel-Carf- } \\
\text { Dex }\end{array}$ & 18 & $4(I-8)$ & $72 \%$ & $\begin{array}{l}\text { Not } \\
\text { reached }\end{array}$ & NR & $\begin{array}{l}\text { Thrombocytopenia }(83 \%) \\
\text { nausea }(67 \%) \text { anemia }(56 \%) \\
\text { fatigue } \\
(50 \%) \text { anorexia }(44 \%) \text { weight } \\
\text { loss }(44 \%) \\
\text { neutropenia }(33 \%)\end{array}$ \\
\hline NCT03II 0562 & BOSTON & III & $\begin{array}{l}\text { Sel-Rev- } \\
\text { Dex } \\
\text { Sel-Vel- } \\
\text { Dex vs } \\
\text { Vel-Dex }\end{array}$ & $\begin{array}{l}24 \\
195 \\
\text { vs } \\
207\end{array}$ & $\begin{array}{l}I(I-8) \\
I-3\end{array}$ & $\begin{array}{l}92 \% \\
76.4 \% \text { vs } \\
62.3 \% \\
(p=0.0012)\end{array}$ & $\begin{array}{l}10.3 \\
13.93 \text { vs } \\
9.46, \mathrm{HR}= \\
0.70 \\
P=0.0066\end{array}$ & $\begin{array}{l}\text { NR } \\
\text { Not } \\
\text { reached vs } \\
25 \\
(p=0.28)\end{array}$ & $\begin{array}{l}\text { Thrombocytopenia }(63 \%) \\
\text { neutropenia }(63 \%) \\
\text { grade } \geq 3 \text { : thrombocytopenia } \\
(35.9 \% \text { vs } 15.2 \%) \text {, fatigue } \\
(11.3 \% \text { vs } 0.5 \%) \text {, nausea } \\
(7.7 \% \text { vs } 0 \%) \text { PN rates } \\
\text { (grade } \geq 2)(2 I .0 \% \text { vs } 34.3 \% \text {, } \\
p=0.00 I 3)\end{array}$ \\
\hline
\end{tabular}

Abbreviations: Sel, selinexor; Vel, bortezomib; Dex, dexamethasone; Dara, daratumumab; Pom, pomalidomide; Rev, lenalidomide; Carf, carfilzomib; NR, not reported; PN, peripheral neuropathy; ORR, overall response rate; PFS, progression-free survival; OS, overall survival.

combination according to disease status, previous exposure to anti-myeloma agents and patient characteristics including age and comorbidities. Well-designed studies are needed in order to address the effect of selinexor in subgroup of patients of special interest such as those with ultra-high risk cytogenetics or extramedullary 
Table 2 Summary of Frequent Selinexor-Induced Toxicities and Management According to Gavriatopoulou et al ${ }^{49}$

\begin{tabular}{|l|l|}
\hline $\begin{array}{l}\text { Close patient } \\
\text { surveillance }\end{array}$ & Weekly for the first 8 weeks: whole blood count, serum sodium, patient weight \\
\hline Nausea and Vomiting & $\begin{array}{l}\text { First } 8 \text { weeks: } 5-\mathrm{HT} 3 \text { receptor antagonists (eg ondansetron } 8 \mathrm{mg} \text { ) before and after selinexor and the day after. } \\
\text { Higher risk for nausea: olanzapine } 5-10 \mathrm{mg} \text { po daily or NKI receptor antagonist (eg rolapitant) }\end{array}$ \\
\hline Diarrhea & Loperamide as per institutional guidelines \\
\hline Thrombocytopenia & $\begin{array}{l}\text { Platelets below } 25,000 / \mathrm{mm}^{3} \rightarrow \text { TPO agonists: romiplostim, } 5-10 \mathrm{mcg} / \mathrm{kg} \text { iv once-weekly or eltrombopag } 50 \mathrm{mg} \text { po } \\
\text { daily }\end{array}$ \\
\hline Neutropenia & ANC below $500 \mathrm{~mm}^{3} \rightarrow$ G-CSF: filgrastim sc or iv, or pegfilgrastim sc \\
\hline $\begin{array}{l}\text { Hyponatremia } \\
\text { Fatigue }\end{array}$ & $\begin{array}{l}\text { Close monitoring of hydration and serum sodium, treatment according to institutional guidelines } \\
\text { Grade } 2 \text { of higher } \rightarrow \text { consider methylphenidate } 10 \mathrm{mg} \text { po daily. }\end{array}$ \\
\hline
\end{tabular}

Abbreviations: NKI, neurokinin-I; TPO, thrombopoietin; ANC, absolute neutrophil count; G-CSF, granulocyte-colony stimulating factor; sc, subcutaneous; iv, intravenously; po, per os.

disease or involvement of the central nervous system or plasma cell leukemia. Preventive measures and close surveillance are key factors in the management of selinexor-related toxicities (Table 2). Ongoing and future studies will determine the exact position of selinexorbased treatments in the therapeutic continuum of patients with MM.

\section{Disclosure}

PM and INS declare no competing interests. MG declares consultancy and honoraria from Amgen, Karyopharm, Genesis Pharma, Janssen and Takeda. ET declares honoraria from BMS, Janssen, Celgene, Takeda, Genesis Pharma, Amgen and Novartis, and research funding from Janssen, Amgen, Takeda and Genesis Pharma.

\section{References}

1. Rajkumar SV. Multiple myeloma: 2020 update on diagnosis, risk-stratification and management. Am J Hematol. 2020;95 (5):548-567. doi:10.1002/ajh.25791

2. Ntanasis-Stathopoulos I, Gavriatopoulou M, Kastritis E, Terpos E, Dimopoulos MA. Multiple myeloma: role of autologous transplantation. Cancer Treat Rev. 2020;82:101929. doi:10.1016/j. ctrv.2019.101929

3. Ntanasis-Stathopoulos I, Gavriatopoulou M, Terpos E. Antibody therapies for multiple myeloma. Expert Opin Biol Ther. 2020;20 (3):295-303. doi:10.1080/14712598.2020.1717464

4. Gavriatopoulou M, Ntanasis-Stathopoulos I, Dimopoulos MA, Terpos E. Anti-BCMA antibodies in the future management of multiple myeloma. Expert Rev Anticancer Ther. 2019;19(4):319-326. doi:10.1080/14737140.2019.1586539

5. Lonial S, Lee HC, Badros A, et al. Belantamab mafodotin for relapsed or refractory multiple myeloma (DREAMM-2): a two-arm, randomised, open-label, phase 2 study. Lancet Oncol. 2020;21(2):207-221. doi:10.1016/S1470-2045(19)30788-0
6. Terpos E; Ntanasis-Stathopoulos I; International Myeloma Society. Multiple myeloma: clinical updates from the american society of hematology annual meeting 2019. Clin Lymphoma Myeloma Leuk. 2020. doi:10.1016/j.clml.2020.03.017

7. Syed YY. Selinexor: first global approval. Drugs. 2019;79 (13):1485-1494. doi:10.1007/s40265-019-01188-9

8. Chen C, Siegel D, Gutierrez M, et al. Safety and efficacy of selinexor in relapsed or refractory multiple myeloma and Waldenstrom macroglobulinemia. Blood. 2018;131(8):855-863. doi:10.1182/ blood-2017-08-797886

9. Etchin J, Sun Q, Kentsis A, et al. Antileukemic activity of nuclear export inhibitors that spare normal hematopoietic cells. Leukemia. 2013;27(1):66-74. doi:10.1038/leu.2012.219

10. Depping R, von Fallois M, Landesman Y, Kosyna FK. The nuclear export inhibitor selinexor inhibits hypoxia signaling pathways and $3 \mathrm{D}$ spheroid growth of cancer cells. Onco Targets Ther. 2019;12:8387-8399. doi:10.2147/OTT.S213208

11. Tai YT, Landesman Y, Acharya C, et al. CRM1 inhibition induces tumor cell cytotoxicity and impairs osteoclastogenesis in multiple myeloma: molecular mechanisms and therapeutic implications. Leukemia. 2014;28(1):155-165. doi:10.1038/leu.2013.115

12. Taylor-Kashton C, Lichtensztejn D, Baloglu E, et al. XPO1 inhibition preferentially disrupts the 3D nuclear organization of telomeres in tumor cells. J Cell Physiol. 2016;231(12):2711-2719. doi:10.1002/jcp.25378

13. Schmidt J, Braggio E, Kortuem KM, et al. Genome-wide studies in multiple myeloma identify XPO1/CRM1 as a critical target validated using the selective nuclear export inhibitor KPT-276. Leukemia. 2013;27(12):2357-2365. doi:10.1038/leu.2013.172

14. Allegra A, Innao V, Allegra AG, Leanza R, Musolino C. Selective inhibitors of nuclear export in the treatment of hematologic malignancies. Clin Lymphoma Myeloma Leuk. 2019;19 (11):689-698. doi:10.1016/j.clml.2019.08.011

15. Terpos E, Ntanasis-Stathopoulos I, Gavriatopoulou M, Dimopoulos MA. Pathogenesis of bone disease in multiple myeloma: from bench to bedside. Blood Cancer J. 2018;8(1):7. doi:10.1038/s41408-017-0037-4

16. Muz B, Azab F, de la Puente P, Landesman Y, Azab AK. Selinexor overcomes hypoxia-induced drug resistance in multiple myeloma. Transl Oncol. 2017;10(4):632-640. doi:10.1016/j.tranon.2017.04.010

17. Vogl DT, Dingli D, Cornell RF, et al. Selective inhibition of nuclear export with oral selinexor for treatment of relapsed or refractory multiple myeloma. $J$ clin oncol. 2018;36(9):859-866. doi:10.1200/ JCO.2017.75.5207 
18. Argueta C, Kashyap T, Klebanov B, et al. Selinexor synergizes with dexamethasone to repress $\mathrm{mTORC} 1$ signaling and induce multiple myeloma cell death. Oncotarget. 2018;9(39):25529-25544. doi:10.18632/oncotarget.25368

19. Jagannath S, Vogl DT, Dimopoulos M-A, Nooka A, Huff C, Moreau P. Phase $2 b$ results of the STORM Study: oral Selinexor plus Low Dose Dexamethasone (Sd) in patients with Penta-Refractory Myeloma (penta-MM). Clin Lymphoma Myeloma Leuk. 2018;18:S249-S250. doi:10.1016/j.clml.2018.07.149

20. Chari A, Vogl DT, Gavriatopoulou M, et al. Oral selinexor-dexamethasone for triple-class refractory multiple myeloma. $N$ Engl J Med. 2019;381 (8):727-738. doi:10.1056/NEJMoa1903455

21. Gavriatopoulou M, Fotiou D, Ntanasis-Stathopoulos I, Kastritis E, Terpos E, Dimopoulos MA. How I treat elderly patients with plasma cell dyscrasias. Aging (Albany NY). 2018;10(12):4248-4268. doi:10.18632/aging.101707

22. Dimopoulos MA, Sonneveld P, Leung N, et al. International myeloma working group recommendations for the diagnosis and management of myeloma-related renal impairment. $J$ clin oncol. 2016;34 (13):1544-1557. doi:10.1200/JCO.2015.65.0044

23. Machlus KR, Wu SK, Vijey P, et al. Selinexor-induced thrombocytopenia results from inhibition of thrombopoietin signaling in early megakaryopoiesis. Blood. 2017;130(9):1132-1143. doi:10.1182/ blood-2016-11-752840

24. Turner JG, Kashyap T, Dawson JL, et al. XPO1 inhibitor combination therapy with bortezomib or carfilzomib induces nuclear localization of IkappaBalpha and overcomes acquired proteasome inhibitor resistance in human multiple myeloma. Oncotarget. 2016;7 (48):78896-78909. doi:10.18632/oncotarget.12969

25. Gasparetto C, Lentzsch S, Schiller G, et al. Deep and durable responses with Selinexor, Daratumumab, and Dexamethasome (SDd) in Patients with Multiple Myeloma (MM) previously exposed to proteasome inhibitors and immunomodulatory drugs: results of Phase 1b study of SDd. Blood. 2018;132(Supplement 1):599. doi:10.1182/blood-2018-99-117201

26. Nishihori T, Baz R. Selective inhibitors of nuclear export (SINEs) in myeloma: breakthrough or bust? Expert Opin Drug Saf. 2020;19 (2):113-115. doi:10.1080/14740338.2020.1713088

27. Cui Y, Turner JG, Dawson JL, et al. The synergistic effect of melphalan and XPO1 inhibition in pre-clinical models of multiple myeloma. Blood. 2016;128(22):5662. doi:10.1182/blood.V128.22.5662.5662

28. Garg M, Kanojia D, Mayakonda A, et al. Selinexor (KPT-330) has antitumor activity against anaplastic thyroid carcinoma in vitro and in vivo and enhances sensitivity to doxorubicin. Sci Rep. 2017;7 (1):9749. doi:10.1038/s41598-017-10325-x

29. Rosebeck S, Alonge MM, Kandarpa M, et al. Synergistic myeloma cell death via novel intracellular activation of caspase-10-dependent apoptosis by carfilzomib and selinexor. Mol Cancer Ther. 2016;15 (1):60-71. doi:10.1158/1535-7163.MCT-15-0488

30. Knopf KB, Duh MS, Lafeuille MH, et al. Meta-analysis of the efficacy and safety of bortezomib re-treatment in patients with multiple myeloma. Clin Lymphoma Myeloma Leuk. 2014;14(5):380-388. doi:10.1016/j.clml.2014.03.005

31. Available from: https://investors.karyopharm.com/news-releases /news-release-details/karyopharm-announces-phase-3-boston-studymeets-primary-endpoint. Accessed June 22, 2020.

32. Dimopoulos M, Delimpasi S, Simonova M, et al. Weekly selinexor, bortezomib, and dexamethasone (SVd) versus twice weekly bortezomib and dexamethasone ( $\mathrm{Vd}$ ) in patients with multiple myeloma (MM) after one to three prior therapies: initial results of the phase III BOSTON study. J Clin Oncol. 2020;38(suppl; abstr 8501):8501. doi:10.1200/JCO.2020.38.15_suppl.8501

33. Jakubowiak AJ, Jasielec JK, Rosenbaum CA, et al. Phase 1 study of selinexor plus carfilzomib and dexamethasone for the treatment of relapsed/refractory multiple myeloma. Br J Haematol. 2019;186 (4):549-560. doi:10.1111/bjh.15969
34. Podar K, Shah J, Chari A, Richardson PG, Jagannath S. Selinexor for the treatment of multiple myeloma. Expert Opin Pharmacother. 2020;21(4):399-408. doi:10.1080/14656566.2019.1707184

35. Chen C, Gasparetto C, White D, et al. SELINEXOR, POMALIDOMIDE, and DEXAMETHASONE (Spd) in PATIENTS with RELAPSED or REFRACTORY MULTIPLE MYELOMA. EHA Library. Chen C. 06/14/19; 266386; PF587; 2019.

36. Gasparetto C, Lipe B, Tuchman S, et al. Once weekly selinexor, carfilzomib, and dexamethasone (SKd) in patients with relapsed/ refractory multiple myeloma (MM). J Clin Oncol. 2020;38 (15_suppl):8530. doi:10.1200/JCO.2020.38.15_suppl.8530

37. Salcedo M, Lendvai N, Mastey D, et al. Phase I study of selinexor, ixazomib, and low-dose dexamethasone in patients with relapsed or refractory multiple myeloma. Clin Lymphoma Myeloma Leuk. 2020;20(3):198-200. doi:10.1016/j.clml.2019.12.013

38. White D, LeBlanc R, Venner C, et al. Safety and efficacy of the combination of selinexor, lenalidomide and dexamethasone (SRd) in patients with relapsed/refractory multiple myeloma (RRMM). Abstract OAB-083 17th IMWG. September 12-15/2019 Boston, MA, USA. 2019.

39. Lokhorst HM, Plesner T, Laubach JP, et al. Targeting CD38 with daratumumab monotherapy in multiple myeloma. $N$ Engl $J$ Med. 2015;373(13):1207-1219. doi:10.1056/NEJMoa1506348

40. Baz R, Zonder J, Shain KH, et al. Phase I/II study of liposomal doxorubicin (DOX) in combination with selinexor (SEL) and dexamethasone (DEX) for relapsed and refractory multiple myeloma (RRMM). Blood. 2017;130(Supplement 1):3095. doi:10.1182/blood. V130.Suppl_1.3095.3095

41. Tariq M, Farooqui A, Faisal M, et al. Efficacy and safety of selinexor for heavily pretreated multiple myeloma treatment - a systematic review. Abstract FP-115 17th IMWG. September 12-15/2019. Boston, MA, USA; 2019.

42. Cohen AD. CAR T cells and other cellular therapies for multiple myeloma: 2018 update. Am Soc Clin Oncol Educa Book Am Soc Clin Oncol Ann Meeting. 2018;38(38):e6-e15. doi:10.1200/ EDBK_200889

43. Xu J, Chen LJ, Yang SS, et al. Exploratory trial of a biepitopic CAR T-targeting B cell maturation antigen in relapsed/refractory multiple myeloma. Proc Natl Acad Sci U S A. 2019;116(19):9543-9551. doi:10.1073/pnas.1819745116

44. Raje N, Berdeja J, Lin Y, et al. Anti-BCMA CAR T-cell therapy bb2121 in relapsed or refractory multiple myeloma. $N$ Engl J Med. 2019;380(18):1726-1737. doi:10.1056/NEJMoa1817226

45. Mikhael J, Noonan KR, Faiman B, et al. Consensus recommendations for the clinical management of patients with multiple myeloma treated with selinexor. Clinical Lymphoma Myeloma and Leukemia. 2020;20(6):351-357. doi:10.1016/j.clml.2019.12.026

46. Kuruvilla J, Savona M, Baz R, et al. Selective inhibition of nuclear export with selinexor in patients with non-Hodgkin lymphoma. Blood. 2017;129(24):3175-3183. doi:10.1182/blood2016-11-750174

47. Abdul Razak AR, Mau-Soerensen M, Gabrail NY, et al. First-inclass, first-in-human Phase I study of selinexor, a selective inhibitor of nuclear export, in patients with advanced solid tumors. $J$ clin oncol. 2016;34(34):4142-4150. doi:10.1200/JCO.2015.65.3949

48. Vergote IB, Lund B, Peen U, et al. Phase 2 study of the Exportin 1 inhibitor selinexor in patients with recurrent gynecological malignancies. Gynecol Oncol. 2020;156(2):308-314. doi:10.1016/j. ygyno.2019.11.012

49. Gavriatopoulou M, Chari A, Chen C, et al. Integrated safety profile of selinexor in multiple myeloma: experience from 437 patients enrolled in clinical trials. Leukemia. 2020. doi:10.1038/s41375-020-0756-6

50. Chakraborty R, Efficace F. Importance of quality of life in early phase clinical trials: a case study of selinexor in multiple myeloma. Br J Haematol. 2020;189(3). doi:10.1111/bjh.16532 
51. Gavriatopoulou M, Fotiou D, Koloventzou U, et al. Vulnerability variables among octogenerian myeloma patients: a single-center analysis of 110 patients. Leuk Lymphoma. 2019;60(3):619-628. doi:10.1080/10428194.2018.1509323
52. Engelhardt M, Dold SM, Ihorst G, et al. Geriatric assessment in multiple myeloma patients: validation of the International Myeloma Working Group (IMWG) score and comparison with other common comorbidity scores. Haematologica. 2016;101(9):1110-1119. doi:10.3324/haematol.2016.148189

\section{Publish your work in this journal}

OncoTargets and Therapy is an international, peer-reviewed, open access journal focusing on the pathological basis of all cancers, potential targets for therapy and treatment protocols employed to improve the management of cancer patients. The journal also focuses on the impact of management programs and new therapeutic

Submit your manuscript here: https://www.dovepress.com/oncotargets-and-therapy-journal agents and protocols on patient perspectives such as quality of life, adherence and satisfaction. The manuscript management system is completely online and includes a very quick and fair peer-review system, which is all easy to use. Visit http://www.dovepress.com/ testimonials.php to read real quotes from published authors. 